\title{
MicroRNA-25 promotes cell proliferation, migration and invasion in glioma by directly targeting cell adhesion molecule 2
}

\author{
GANG PENG, YI LIU, CHENXING YANG and CHENFU SHEN
}

Department of Neurosurgery, Xiangya Hospital, Central South University, Changsha, Hunan 410008, P.R. China

Received April 14, 2018; Accepted August 19, 2020

DOI: $10.3892 /$ etm.2021.10938

\begin{abstract}
Numerous microRNAs (miRNAs/miRs) have been demonstrated to serve oncogenic or suppressive roles in glioma. Exploration of the underlying molecular mechanism of miRNAs in the development and progression of glioma is beneficial for the identification of novel therapeutic targets. In the present study, the function of miR-25 in glioma progression, as well as its underlying mechanism, were investigated. It was determined that miR-25 was significantly upregulated in glioma tissues and cell lines compared with normal brain tissues and cells, respectively. Furthermore, high expression levels of miR-25 were associated with an advanced clinical stage. The knockdown of miR-25 expression significantly reduced glioma cell proliferation, migration and invasion. Cell adhesion molecule 2 (CADM2) was identified as a direct target of miR-25 in glioma cells. Moreover, CADM2 expression level was significantly downregulated and inversely correlated with miR-25 expression level in glioma tissues, indicating that the expression of CADM2 was negatively regulated by miR-25. The inhibition of CADM2 expression counteracted the effects on glioma cell proliferation, migration and invasion caused by miR-25 downregulation. Furthermore, CADM2 knockdown considerably promoted the proliferation and migration of glioma cells. In summary, the present study demonstrated that miR-25 was significantly upregulated in glioma and that it promoted glioma cell proliferation, migration and invasion, at least partially, by directly targeting CADM2. These findings expanded the understanding of the molecular mechanism that underlies glioma progression.
\end{abstract}

\section{Introduction}

Glioma is the most common primary brain tumor worldwide (1). Despite efforts to facilitate its early detection, as

Correspondence to: Professor Chenfu Shen, Department of Neurosurgery, Xiangya Hospital, Central South University, 87 Xiangya Road, Changsha, Hunan 410008, P.R. China

E-mail: scf680524@126.com

Key words: glioma, microRNA-25, cell adhesion molecule 2, proliferation, migration well as the use of surgical resection combined with radiotherapy and chemotherapy, the majority of patients with glioma are diagnosed at a late stage and their prognosis is poor $(2,3)$. In the last decade, the molecular alterations that occur during glioma progression have been the focus numerous studies and several oncogenes and tumor suppressors have been identified (3-10). An improved understanding of the underlying molecular mechanisms of glioma progression would be beneficial for the identification of novel therapeutic targets.

MicroRNAs (miRNAs/miRs) are endogenous single-stranded non-coding RNA molecules comprising 18-25 nucleotides (11). They are able to bind complementary sites within the 3'-untranslated regions (3'-UTRs) of target genes, suppressing translation or promoting mRNA degradation (11). Since a single miRNA can regulate the expression of numerous target genes, miRNAs serve as key regulators of various physiological and pathological processes, including cell survival, differentiation, proliferation, apoptosis, metabolism and tumorigenesis $(12,13)$. In previous years, various studies have reported deregulation of miRNAs in various types of human cancer, including glioma $(14,15)$. Furthermore, numerous miRNAs have been demonstrated to serve promotional or tumor suppressive roles in glioma. For instance, miR-365 has been demonstrated to inhibit proliferation, migration and invasion in glioma by targeting PIK3R3 (16), whereas miR-599 does so by targeting periostin (17).

Previously, the aberrant expression of miR-25 was indicated to serve tumor promoting and suppressive roles in different types of human cancer, including liver cancer (18). miR-25 was also revealed to enhance cell migration and the invasiveness of non-small-cell lung cancer cells through the MAPK signalling pathway by inhibiting Krüppel-like factor 4 (19). Additionally, miR-25 contributed to cisplatin resistance in gastric cancer cells by inhibiting forkhead box O3a (20). Moreover, miR-25 may promote glioblastoma cell proliferation and invasiveness by directly targeting neurofilament light polypeptide (21). Zhang et al (22) reported that miR-25 promoted glioma cell proliferation by targeting CDK inhibitor $1 \mathrm{C}$ (CDKN1C). These results indicated that miR-25 may serve an oncogenic role in glioma. However, the function of miR-25 in glioma cell migration and its underlying mechanism remain unknown. Therefore, the present study aimed to investigate the function and underlying mechanism of miR-25 in the progression of glioma. 


\section{Materials and methods}

Tissue samples. The present study was approved by the Ethics Committee of Xiangya Hospital of Central South University (Changsha, China). A total of 15 normal brain tissues and 53 primary glioma tissues were obtained between March 2010 and May 2014. Patients were included in the current study if they were diagnosed with primary glioma that was confirmed by an independent pathologist at Xiangya Hospital. Patients were excluded if patients with glioma had received chemotherapy or radiotherapy prior to surgery. The normal brain tissues were collected from 15 patients without malignancy who underwent surgery to reduce increased intracranial pressure or to treat a severe head injury. The histomorphology of these tissues was confirmed by the hospital's Department of Pathology. These 15 patients included 9 males and 6 females (age range, 43-66 years; mean age, 55.1 years) and the 53 patients with glioma included 31 males and 22 females (age range, 34-68 years; mean age, 53.5 years). Glioma was pathologically confirmed and was staged according to the TNM classification. Written informed consent was obtained from all participants.

Cell culture. Human glioma cell lines (U-373MG Uppsala, U-87MG Uppsala, U251MG and T98G) were obtained from the Cell Bank of the Chinese Academy of Sciences. Human normal brain cells were purchased from ScienCell Research Laboratories, Inc. The cells were cultured in DMEM (Thermo Fisher Scientific, Inc.) supplemented with 10\% FBS (Thermo Fisher Scientific, Inc.) at $37^{\circ} \mathrm{C}$ in a humidified incubator with $5 \% \mathrm{CO}_{2}$.

Transfection. Cell transfection was performed using Lipofectamine 2000 (Thermo Fisher Scientific, Inc.), according to the manufacturer's protocol. T98G and U251MG cells $(100,000$ cells/well) were transfected with $100 \mathrm{nM}$ negative control (NC) inhibitors (cat. no. NCSTUD001; Sigma-Aldrich; Merck KGaA), miR-25 inhibitors (cat. no. HSTUD0414; Sigma-Aldrich; Merck KGaA), miR-NCs (cat. no. HMC0002; Sigma-Aldrich; Merck KGaA), miR-25 mimics (cat. no. HMI0414; Sigma-Aldrich; Merck KGaA), NC small interfering (si)RNA (siNC; cat. no. sc-37007; Santa Cruz Biotechnology, Inc.) or cell adhesion molecule 2 (CADM2) siRNA (siCADM2; cat. no. sc-78228; Santa Cruz Biotechnology Inc.). The sequences used for all transfection experiments were not commercially available. After $48 \mathrm{~h}$ of transfection, subsequent experiments were performed as described below.

Reverse transcription-quantitative PCR (RT-qPCR). Total RNA was isolated from tissues or T98G and U251MG cells using TRIzol ${ }^{\circledR}$ reagent (Thermo Fisher Scientific, Inc.). The total RNA was then reverse transcribed using PrimeScript II 1st Strand cDNA Synthesis kit (Takara Bio, Inc.), according to the manufacturer's protocol. The cDNA was amplified by qPCR using SensiFAST ${ }^{\text {TM }}$ SYBR $^{\circledR}$ Hi-ROX Mix (Bioline), with U6 and GAPDH as internal references to normalize expression data. The thermocycling conditions were as follows: Initial denaturation at $95^{\circ} \mathrm{C}$ for $3 \mathrm{~min} ; 40$ cycles at $95^{\circ} \mathrm{C}$ for $30 \mathrm{sec}$ and $60^{\circ} \mathrm{C}$ for $30 \mathrm{sec}$. The primer sequences were as follows:
miR-25 forward, 5'-CTGGTAGGCATTGCACTTGTCT-3' and reverse, 5'-TCAACTGGTGTCGTGGAG-3'; U6 forward, 5'-CTCGCTTCGGCAGCACATATACT-3' and reverse, 5'-CGCTTCACGAATTTGCGTGT-3'; GAPDH forward, 5'-CTGGGCTACACTGAGCACC-3' and reverse, 5'-AAG TGGTCGTTGAGGGCAATG-3'; CADM2 forward, 5'-AAA CTTCCAAGGCATATCTCACC-3' and reverse, 5'-TGCGAT TTGCATCCTCTTCTT-3'. Relative gene expression levels were analyzed according to the $2^{-\Delta \Delta C q}$ method (23).

Cell Counting Kit-8 (CCK-8) assay. A CCK-8 assay was used to determine the cell proliferation rate. The transfected T98G and U251MG cells were seeded $\left(5 \times 10^{3}\right.$ cells/well) into 96-well plates and $20 \mu \mathrm{l}$ CCK-8 reagent (Beyotime Institute of Biotechnology) was added to each well. The cells were incubated in a humidified incubator at $37^{\circ} \mathrm{C}\left(5 \% \mathrm{CO}_{2}\right)$ for 24,48 and $72 \mathrm{~h}$ time intervals $(14,15)$. The optical density at a $450 \mathrm{~nm}$ absorbance was determined using a microplate reader.

Wound healing analysis. Transfected T98G and U251MG cells were seeded in $24-$ well plates and cultured at $37^{\circ} \mathrm{C}$ until $\sim 90 \%$ confluence. A wound was scratched across the center of each well using a $10 \mu 1$ sterile pipette tip. The cells were then washed twice with PBS and cultured in serum-free DMEM at $37^{\circ} \mathrm{C}$ for $24 \mathrm{~h}$. Images were captured under a light microscope (magnification, $\mathrm{x} 40$ ) at 0 and 24 -h time points.

Transwell assay. For the Transwell assay, 24-well Transwell chambers (8-mm pore size; Corning Inc.) pre-coated with Matrigel Basement Membrane Matrix (BD Biosciences) at room temperature for $1 \mathrm{~h}$ were used. Briefly, DMEM with $10 \%$ FBS was added to the lower chambers and transfected T98G and U251MG cells ( $1 \times 10^{4}$ cells $\left./ \mathrm{ml}\right)$ in 300 ul serum-free DMEM were added to the upper chamber. Following incubation at $37^{\circ} \mathrm{C}$ for $24 \mathrm{~h}$, the cells on the upper surface of the insert were removed using a cotton-tipped swab. The invaded cells were then fixed in 4\% paraformaldehyde (Sigma-Aldrich; Merck KGaA) for 20 min and stained with $0.5 \%$ crystal violet (Sigma-Aldrich; Merck KGaA) for 5 min at room temperature and images were captured under an inverted light microscope (magnification, x200; Olympus Corporation).

Bioinformatics analysis. TargetScan 7.1 software (www. targetscan.org) was used to analyze the putative target genes of miR-25. The resultant data revealed that CADM2 was a putative target gene of miR-25 and as such was selected in the search for further mutual verification.

Luciferase reporter assay. The wild-type (WT) and mutant (MT) 3'-UTR sequences of CADM2 were synthesized by Shanghai GenePharma Co., Ltd. and cloned into PGL3 luciferase reporter plasmids (Ambion; Thermo Fisher Scientific, Inc.). T98G and U251MG cells (100,000 cells/well) seeded in 24-well plates were transfected with $100 \mathrm{nM}$ WT or MT luciferase reporter plasmids, and either $100 \mathrm{nM}$ miR-25 or miR-NC using Lipofectamine ${ }^{\circledR} 2000$. The sequences used were mentioned previously. After $24 \mathrm{~h}$, the firefly and Renilla luciferase activities were detected using the Luc-Pair miRNA Luciferase Assay kit (GeneCopoeia, Inc.), according to the manufacturer's instructions. 
Table I. Association between miR-25 expression level and clinicopathological characteristics of patients with glioma.

\begin{tabular}{|c|c|c|c|c|}
\hline Variable & $\begin{array}{c}\text { Number of } \\
\text { patients }(n=53)\end{array}$ & $\begin{array}{c}\text { Low miR-25 } \\
\text { expression }(n=27)\end{array}$ & $\begin{array}{c}\text { High miR-25 } \\
\text { expression }(n=26)\end{array}$ & P-value \\
\hline Age & & & & 0.275 \\
\hline$<50$ years & 28 & 12 & 16 & \\
\hline$\geq 50$ years & 25 & 15 & 10 & \\
\hline Sex & & & & 0.583 \\
\hline Male & 31 & 17 & 14 & \\
\hline Female & 22 & 10 & 12 & \\
\hline TNM stage & & & & 0.028 \\
\hline I-II & 29 & 19 & 10 & \\
\hline III-IV & 24 & 8 & 16 & \\
\hline
\end{tabular}

miR, microRNA.

A

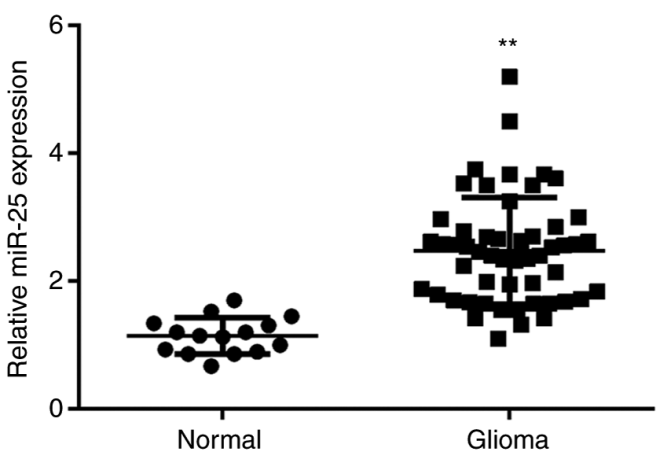

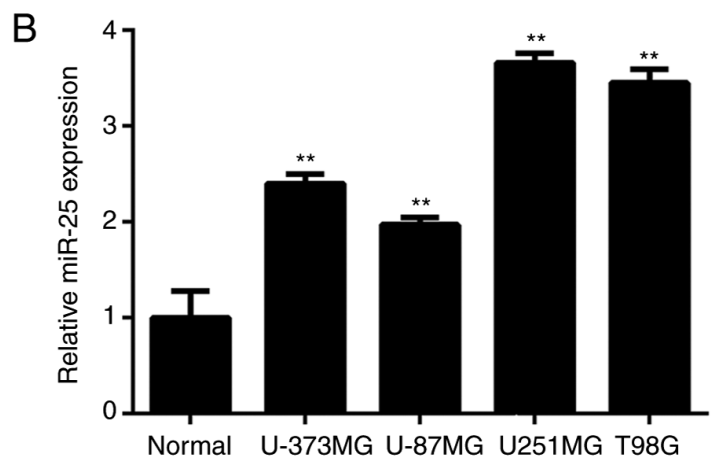

Figure 1. miR-25 is upregulated in glioma. (A) miR-25 expression levels were determined in glioma tissues and normal brain tissues using RT-qPCR. (B) RT-qPCR was used to measure miR-25 expression level in glioma cell lines and normal brain tissues. ${ }^{* *} \mathrm{P}<0.01$ vs. Normal. miR, microRNA; RT-qPCR, reverse transcription-quantitative PCR.

Western blot analysis. Total protein was extracted from cell lines using RIPA lysis buffer (Thermo Fisher Scientific, Inc.). The protein concentration was determined using a bicinchoninic acid assay kit (Thermo Fisher Scientific, Inc.), according to the manufacturer's protocol. The proteins $(50 \mu \mathrm{g} / \mathrm{lane})$ were separated by SDS-PAGE using 10\% gels and transferred to PVDF membranes (Thermo Fisher Scientific, Inc.). The membranes were blocked in 5\% skimmed milk at room temperature for $3 \mathrm{~h}$ and subsequently washed three times with PBS and $0.05 \%$ Tween-20 (PBST) prior to incubation with rabbit anti-human CADM2 (1:300 dilution; cat. no. ab64873; Abcam) or rabbit anti-human GAPDH antibodies (1:500 dilution; cat. no. ab9485; Abcam) at room temperature for $3 \mathrm{~h}$. After three washes in PBST, the membranes were incubated with an HRP-conjugated goat anti-rabbit secondary antibody (1:5,000 dilution; cat. no. ab7090; Abcam) at room temperature for $1 \mathrm{~h}$. The blots were visualized using Western Blotting Luminol reagent (Santa Cruz Biotechnology, Inc.) and protein expression was semi-quantified using Image-Pro Plus software 6.0 (Media Cybernetics, Inc.). GAPDH was used for the normalization of protein expression levels.

Statistical analysis. Experiments were performed in triplicate. Prism 5 software (GraphPad Software, Inc.) was used for all statistical analyses and the data are presented as the mean \pm standard deviation. An unpaired Student's t-test was used to analyze the differences between two groups, whilst one-way ANOVA followed by Tukey's post-hoc test was used to analyze differences among multiple groups. $\chi^{2}$ test analysis was conducted to determine the association between miR-25 expression levels and the clinicopathological features of glioma. Spearman's rank correlation was used to analyze the correlation between the expression levels of CADM2 and miR-25 in glioma tissues. $\mathrm{P}<0.05$ was considered to indicate a statistically significant difference.

\section{Results}

miR-25 is upregulated in glioma. In the present study, RT-qPCR was conducted to determine miR-25 expression levels in glioma. The expression level of miR-25 was significantly upregulated in glioma tissues compared with normal brain tissues (Fig. 1A). The patients with glioma were subsequently divided into high and low miR-25-expression groups according to the mean miR-25 expression level. As presented in Table I, a high expression level of miR-25 was significantly associated with advanced clinical stage in patients with glioma. Additionally, miR-25 expression was significantly 
A

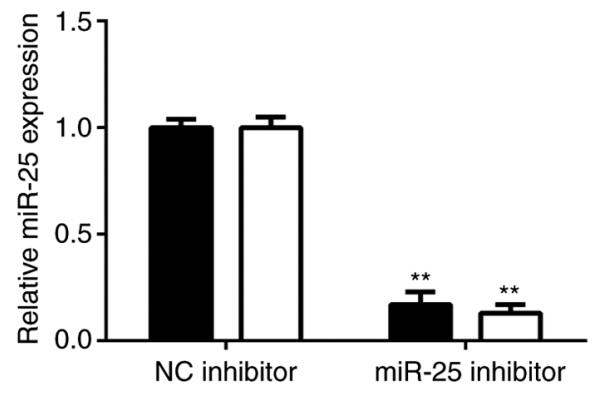

C

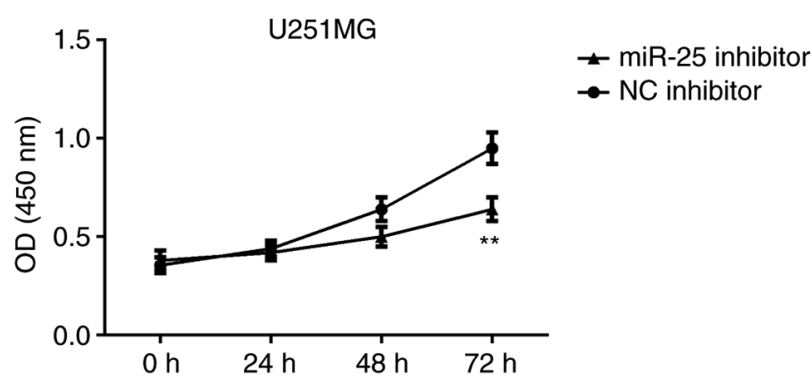

E

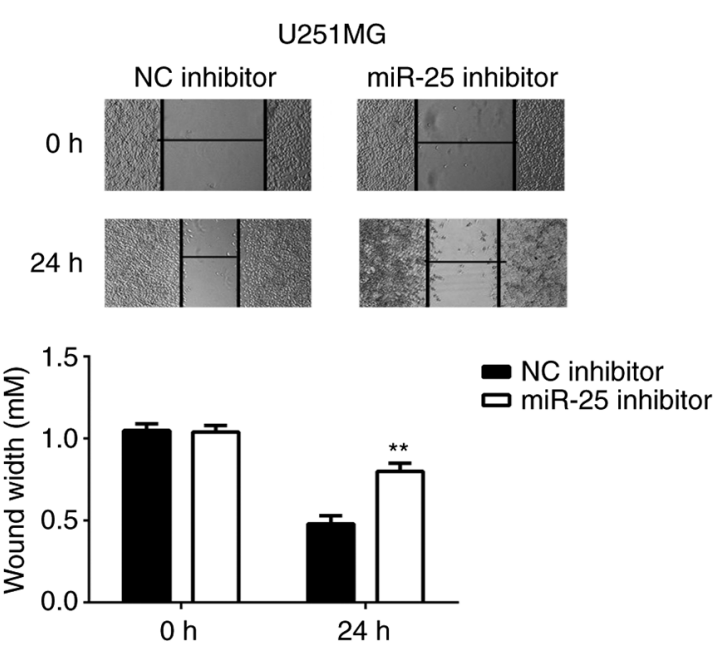

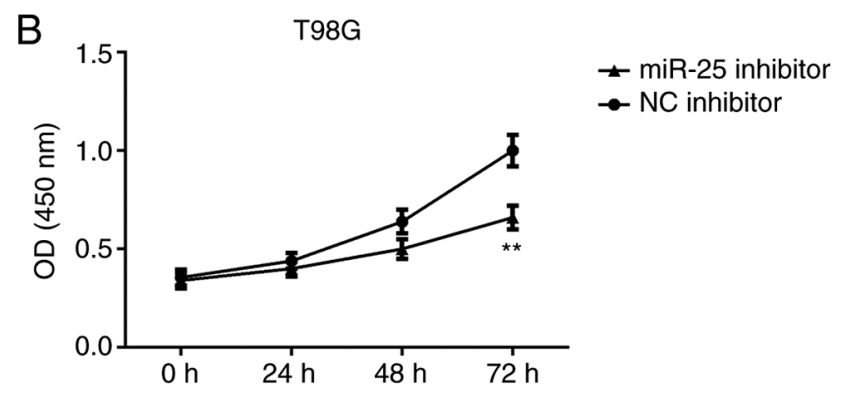

T98G

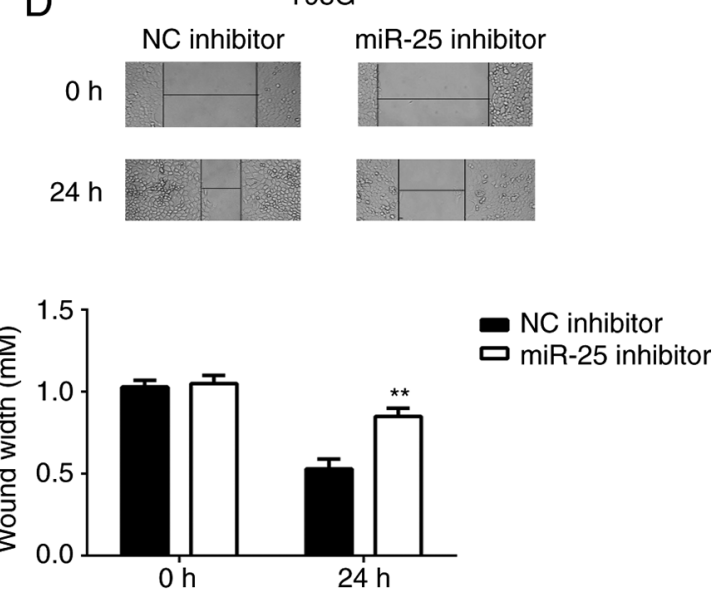

$\mathrm{F}$

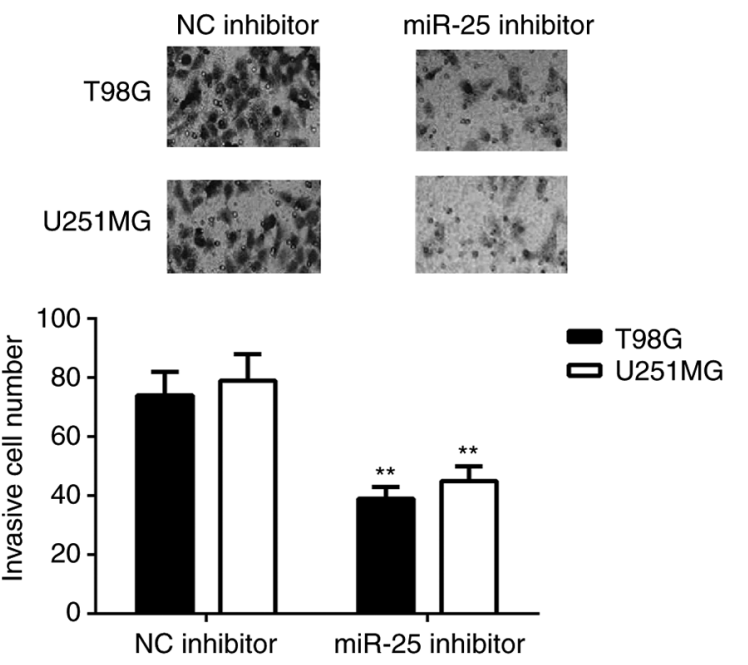

Figure 2. Knockdown of miR-25 inhibits the proliferation and migration of glioma cells. miR-25 inhibitor or NC inhibitor were transfected into T98G and U251MG cells. (A) Following transfection, reverse transcription-quantitative PCR was used to measure the expression of miR-25. The Cell Counting Kit-8 assay was used to examine the proliferation of (B) T98G and (C) U251MG cells. Wound healing assays were used to assess (D) T98G and (E) U251MG cell migration. (F) Cell invasion was determined using Transwell assays. ${ }^{* *} \mathrm{P}<0.01$ vs. NC inhibitor. miR, microRNA; NC, negative control; OD, optical density. Magnification for cell migration, x40. Magnification for cell invasion, x200.

increased in glioma cell lines compared with normal brain tissue cells (Fig. 1B).

Knockdown of miR-25 suppresses the proliferation, migration and invasiveness of glioma cells. The function of miR-25 in glioma cells was subsequently investigated. To knock down miR-25 expression, U251MG and T98G cells were transfected with an miR-25 inhibitor. These cell lines were chosen for subsequent experiments as they exhibited the highest expression levels of miR-25. Cells that were transfected with the NC inhibitor served as the control group. The miR-25 expression level was significantly downregulated in T98G and U251MG cells transfected with miR-25 inhibitor compared with the control group (Fig. 2A). As presented in Fig. 2B-C, CCK-8 assay data showed that the inhibition of miR-25 significantly reduced the proliferation rate of $\mathrm{U} 251 \mathrm{MG}$ and $\mathrm{T} 98 \mathrm{G}$ cells at $72 \mathrm{~h}$ post-transfection. Wound healing and Transwell assays were then performed to investigate the role of miR-25 in glioma cell migration and invasion. As demonstrated by Fig. 2D-F, downregulation of miR-25 markedly reduced the migratory and invasive capacities of U251MG and T98G cells, indicated that the knockdown of miR-25 inhibited the proliferation and migration of glioma cells. 
A WT CADM2 3'UTR $\quad 5^{\prime} \ldots$ UUGAAAUAUACUCAGUGCAAUA....3'

\|\|\|\|

miR-25 $3^{\prime} \ldots$..AGUCUGGCUCUGUUCACGUUAC.... $5^{\prime}$

MT CADM2 3'UTR $\quad 5^{\prime} \ldots$ UUGAAAUAUACUCAGCGUUUA....3'

$\mathrm{B}$

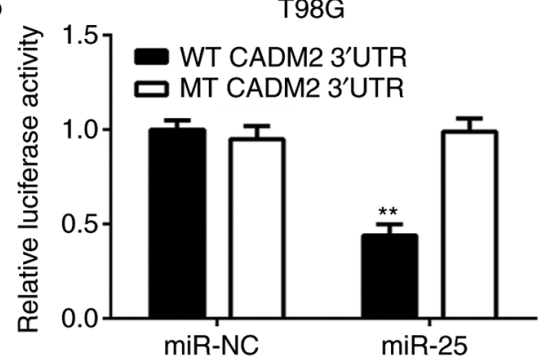

C

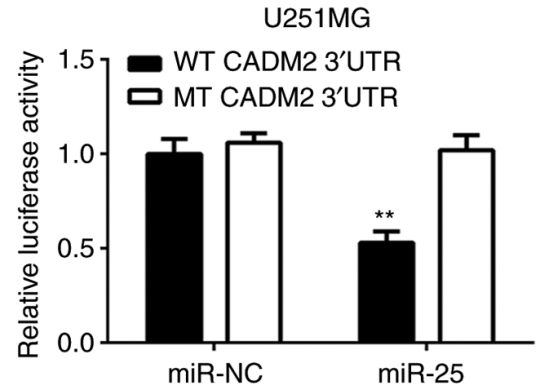

D

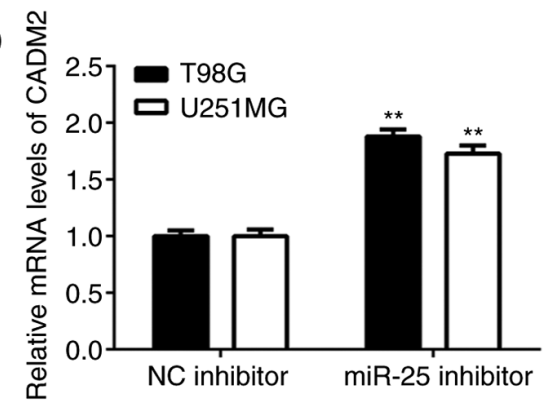

E

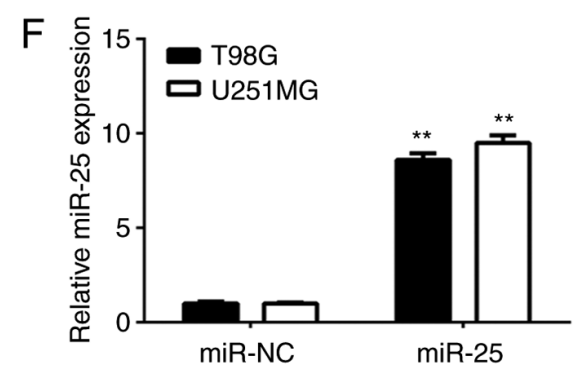

$\mathrm{H}$

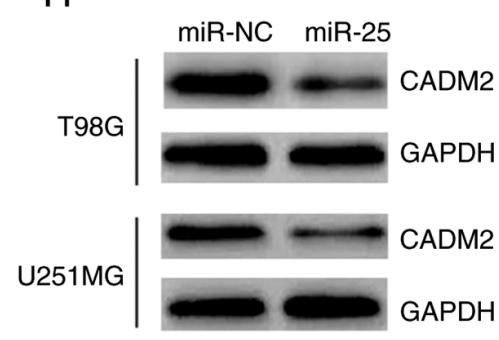

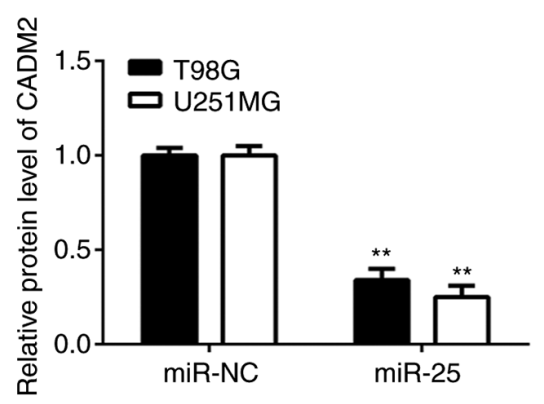

Figure 3. CADM2 is a target gene of miR-25 in glioma. (A) Putative WT and MT binding sequences in the 3'-UTR of CADM2. (B) T98G and (C) U251MG cells were co-transfected with miR-25 mimics or miR-NC and WT-CADM2 or MT-CADM2 reporter gene plasmids. Following transfection for $48 \mathrm{~h}$, dual luciferase reporter assays were conducted. ${ }^{* *} \mathrm{P}<0.01 \mathrm{vs.} \mathrm{miR-NC}$. The (D) mRNA and (E) protein expression levels of CADM2 were detected using RT-qPCR and western blotting, respectively, in T98G and U251MG cells transfected with miR-25 inhibitor or NC inhibitor. ${ }^{* *} \mathrm{P}<0.01$ vs. NC inhibitor. The expression levels of (F) miR-25 and (G and H) CADM2 were detected using RT-qPCR and western blotting in T98G and U251MG cells transfected with miR-25 mimics or miR-NC. ** $\mathrm{P}<0.01$ vs. miR-NC. CADM2, cell adhesion molecule 2; miR, microRNA; MT, mutant type; NC, negative control; RT-qPCR, reverse transcription-quantitative PCR; UTR, untranslated region; WT, wild-type.

CADM2 is a target of miR-25 in glioma cells. Bioinformatics analysis was performed to predict the potential target genes of miR-25. The data revealed that CADM2 was a putative target gene of miR-25 (Fig. 3A) and luciferase reporter gene analysis verified this prediction. The overexpression of miR-25 significantly inhibited the luciferase activity of the WT 3'-UTR of 
A

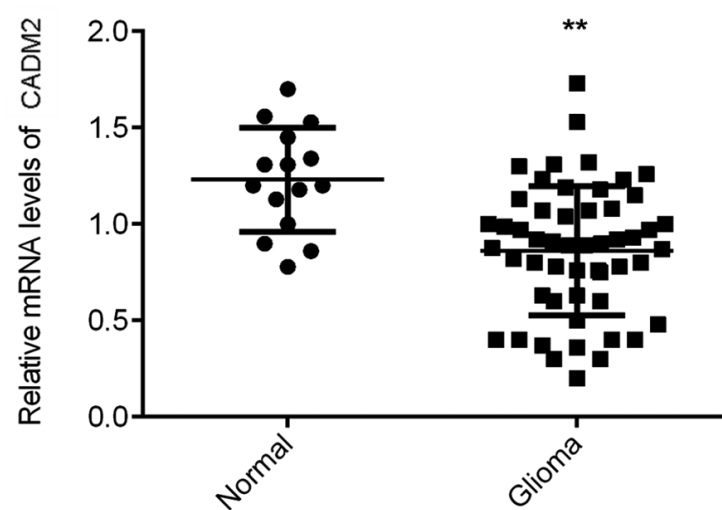

C

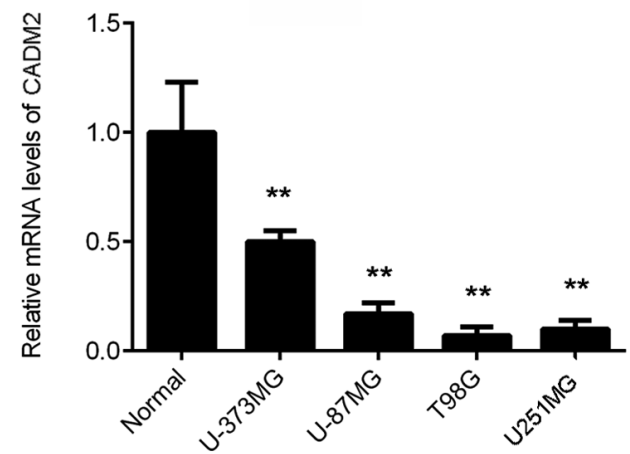

B

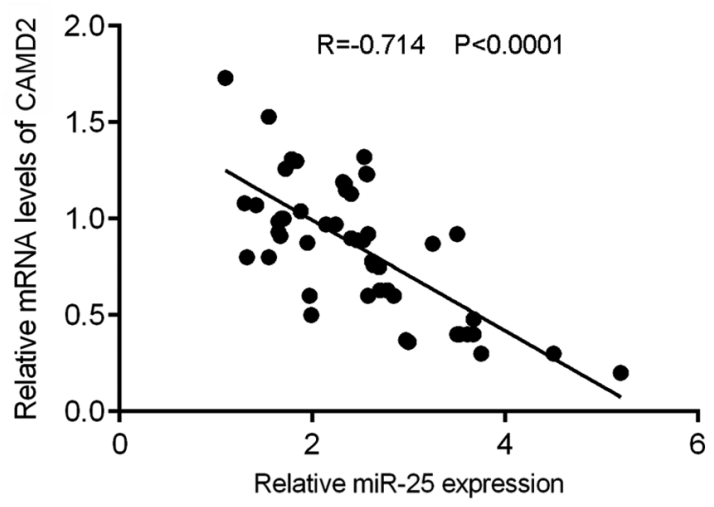

D

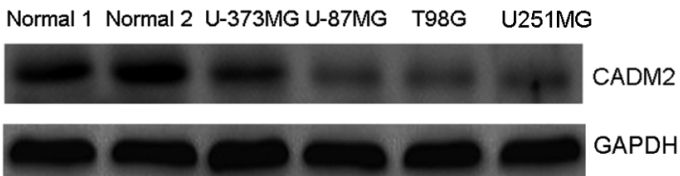

Figure 4. CADM2 is downregulated in glioma. (A) The mRNA expression level of CADM2 was determined in glioma tissues and normal brain tissues using RT-qPCR. (B) miR-25 expression was inversely correlated with CADM2 mRNA expression level in glioma tissues. The (C) mRNA and (D) protein expression levels of CADM2 were detected using RT-qPCR and western blotting, respectively, in glioma cell lines and normal brain tissues. ${ }^{* *} \mathrm{P}<0.01$ vs. Normal. CADM2, cell adhesion molecule 2; miR, microRNA; RT-qPCR, reverse transcription-quantitative PCR; normal 1 and 2, two different normal brain tissues obtained from two controls.

CADM2, but not that of the MT 3'-UTR in T98G and U251MG cells (Fig. 3B and C). Additionally, it was revealed that miR-25 knockdown significantly increased the mRNA and protein expression levels of CADM2 in both cell lines (Fig. 3D and E). By contrast, transfection with miR-25 mimics significantly enhanced the expression of miR-25 and reduced the expression level of CADM2 in glioma cells (Fig. 3F-H). Taken together, these findings suggested that CADM2 is a direct target gene of miR-25 in glioma cells.

CADM2 is downregulated in glioma. The expression of CADM2 in glioma was also determined. RT-qPCR results demonstrated that CADM2 was significantly downregulated in glioma tissues compared with normal brain tissues (Fig. 4A). Notably, the CADM2 expression level was negatively correlated with that of miR-25 in glioma tissues (Fig. 4B). Consistently, the mRNA and protein levels of CADM2 were also significantly lower in glioma cell lines compared with normal brain tissues (Fig. 3C and D, respectively). Thus, CADM2 was downregulated in glioma, which may be due to the upregulation of miR-25.

Knockdown of CADM2 reverses the inhibitory effects of miR-25 downregulation on the proliferation, migration and invasion of glioma cells. Based on the aforementioned findings, it was hypothesized that CADM2 may be involved in the miR-25-mediated proliferation and migration of glioma cells. To test this hypothesis, T98G and U251MG cells were transfected with NC siRNA or siCADM2. After transfection, the protein expression levels of CADM2 were significantly reduced in the siCADM2 group compared with the NC siRNA group (Fig. 5A). CCK-8 and wound healing assays were performed to assess cell proliferation and migration. As shown in Fig. 5B-E, the knockdown of CADM2 significantly increased the proliferation and migration of glioma cells. These findings suggested that CADM2 serves a suppressive role in glioma cell proliferation and migration.

After that, T98G and U251MG cells were co-transfected with a miR-25 inhibitor and CADM2 siRNA or NC siRNA. Following transfection, the CADM2 mRNA and protein expression levels were significantly reduced in the miR-25 inhibitor + siCADM2 group compared with the miR-25 inhibitor + siNC group (Fig. 6A and B). Data from the CCK-8, wound healing and Transwell assays further demonstrated that the proliferation (at $72 \mathrm{~h}$ ), migration and invasiveness of glioma cells were increased in the miR-25 inhibitor + siCADM2 group compared with the miR-25 inhibitor + siNC group (Fig. 6C-G), suggesting that the knockdown of CADM2 reversed the inhibitory effects of miR-25 downregulation on the proliferation, migration and invasiveness of glioma cells.

\section{Discussion}

The present study aimed to investigate the underlying molecular mechanism of miR-25 in the progression of 
A T98G

U251MG

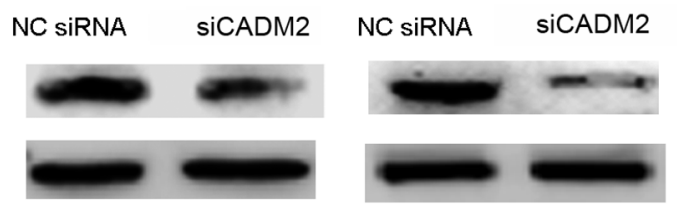

CADM2

GAPDH

B

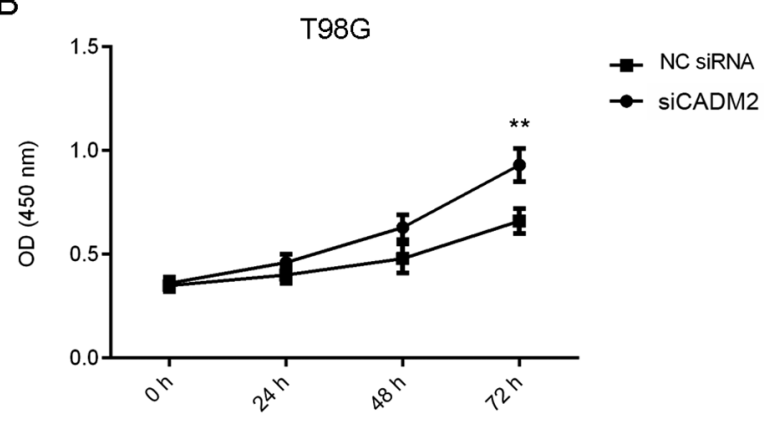

D T98G
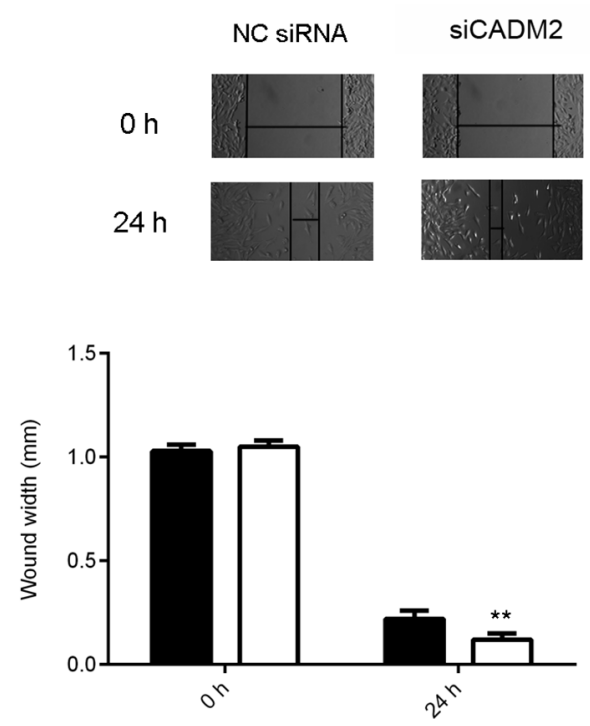

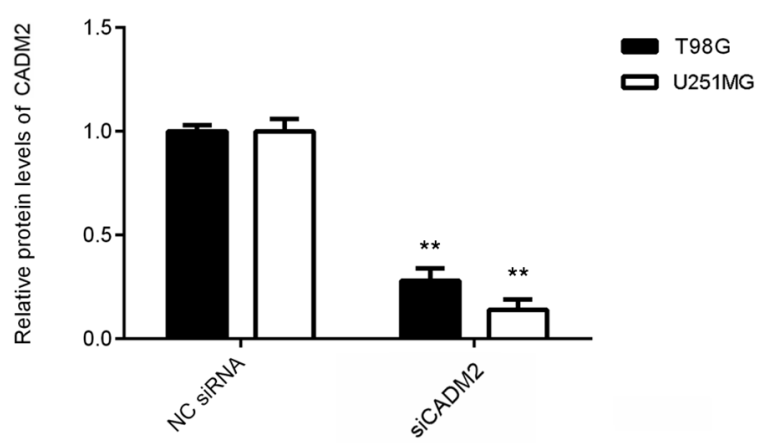

C

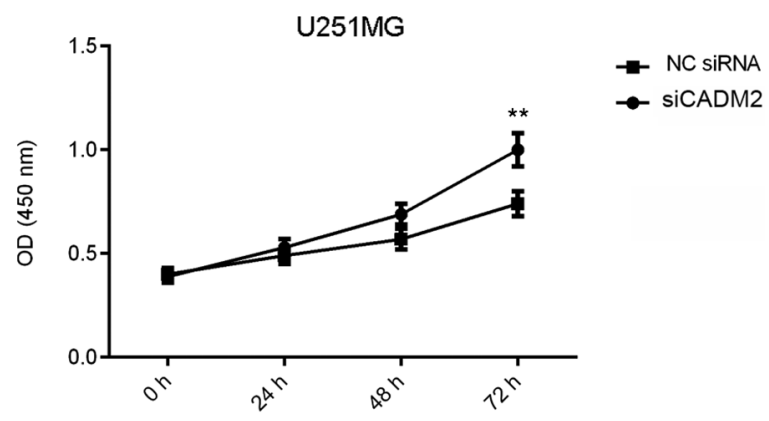

E

U251MG
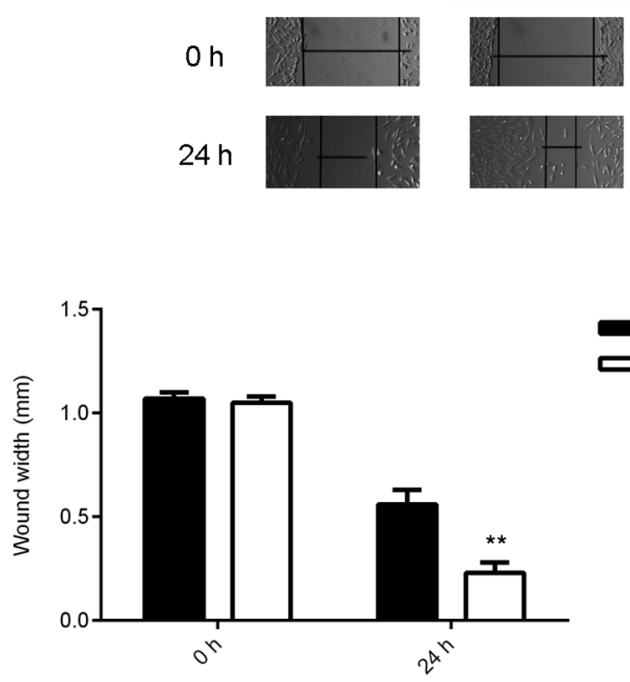

Figure 5. Knockdown of CADM2 significantly increases the proliferation and migration of glioma cells. T98G and U251MG cells were transfected with siCADM2 or NC siRNA. (A) After transfection, the protein levels of CADM2 were examined using western blotting. Cell Counting Kit-8 assay was used to determine cell proliferation of (B) T98G and (C) U251MG cells. Wound healing assay was used to study (D) T98G and (E) U251MG cell migration. ${ }^{* *} \mathrm{P}<0.01$ vs. NC siRNA. CADM2, cell adhesion molecule 2; NC, negative control; si, small interfering RNA. Magnification, $\mathrm{x} 40$.

glioma. The expression of miR-25 was significantly upregulated in glioma tissues and cell lines, and a high expression level of miR-25 was associated with advanced clinical stage. The knockdown of miR-25 expression significantly reduced glioma cell proliferation, migration and invasion. CADM2 was identified as a direct target of miR-25 in glioma cells. Moreover, the expression of level CADM2 was significantly reduced in glioma tissues and was inversely correlated with miR-25 expression. Furthermore, the expression of CADM2 was negatively regulated by miR-25 in glioma cells and
CADM2 knockdown reversed the effects of miR-25 inhibition and promoted glioma cell proliferation, migration and invasion.

An increasing number of studies have reported that the expression levels of miR-25 are frequently upregulated in numerous common types of human cancer and that miR-25 serves an oncogenic role by promoting the malignant phenotype of tumor cells. For example, Xiang et al (24) demonstrated that miR-25 was upregulated in non-small cell lung cancer (NSCLC) tissues and cell lines, and that 
A

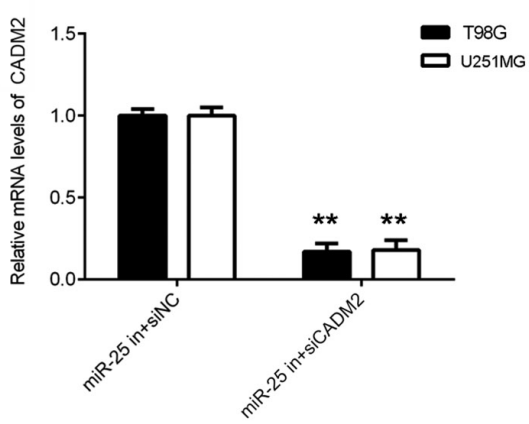

C

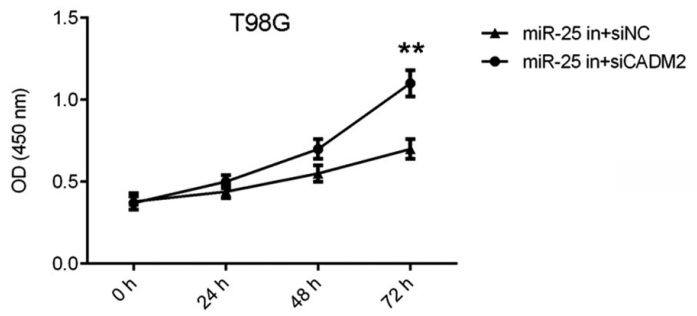

$E$
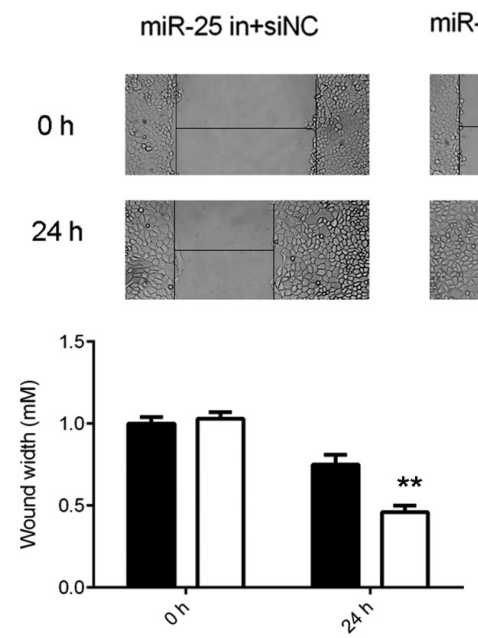

G

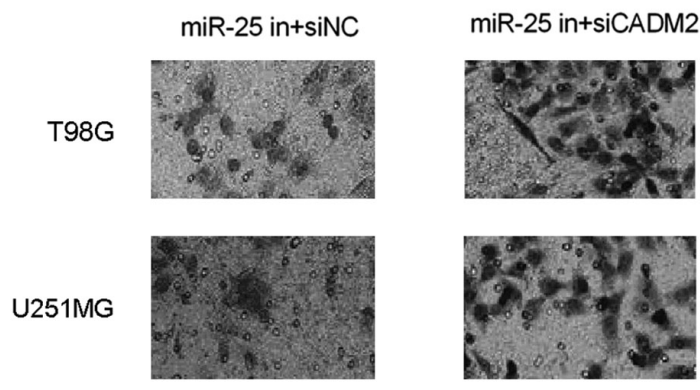

B miR-25 in+siNC
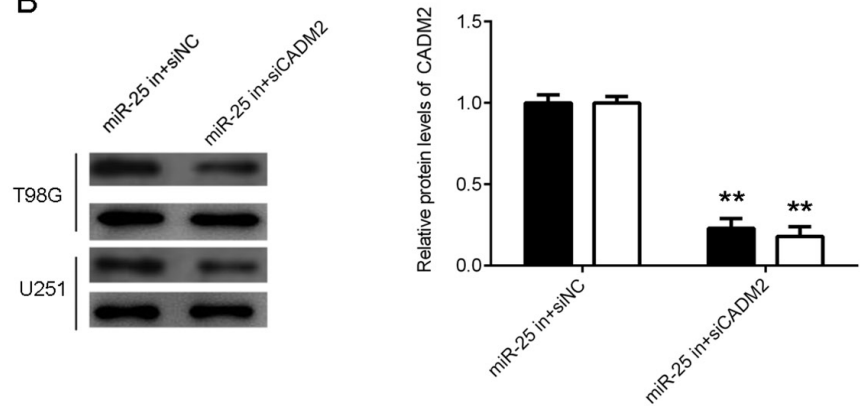

D

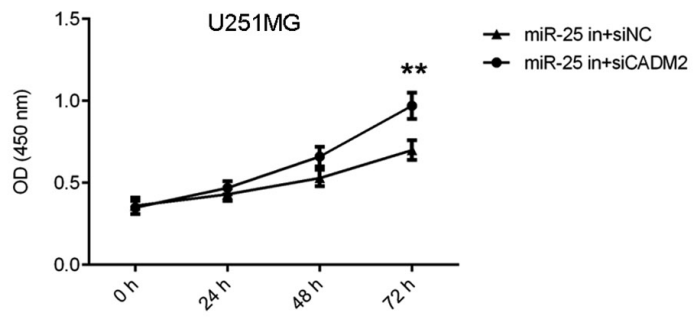

$\mathrm{F}$

U251MG
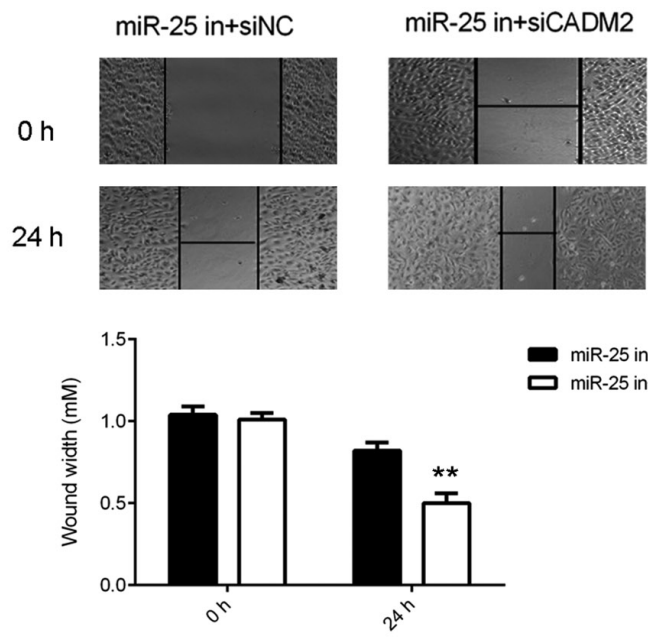

miR-25 in+sinc DiR-25 in+siCADM2

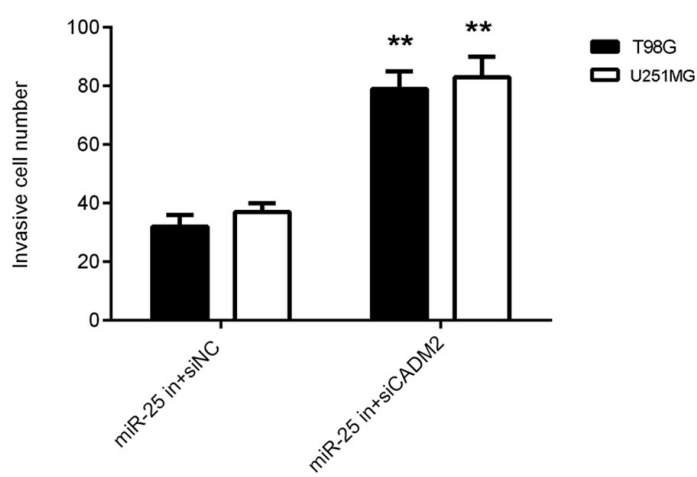

Figure 6. Knockdown of CADM2 rescues the inhibitory effects of miR-25 downregulation on the proliferation and migration of glioma cells. T98G and U251MG cells were co-transfected with miR-25 and siCADM2 or siNC. Following transfection, the (A) mRNA and (B) protein expression levels of CADM2 were examined using reverse transcription-quantitative PCR and western blotting, respectively. The Cell Counting Kit-8 assay was used to determine cell proliferation in (C) T98G and (D) U251MG cells. Wound healing assays were used to study migration in (E) T98G and (F) U251MG cells. (G) Cell invasion was determined using Transwell assays. ${ }^{* *} \mathrm{P}<0.01$ vs. miR-25 in + siNC. CADM2, cell adhesion molecule 2; miR, microRNA; si, small interfering RNA; NC, negative control; in, inhibitor. Magnification for cell migration, x40. Magnification for cell invasion, x200.

this promoted NSCLC cell proliferation and motility by suppressing the expression of F-box/WD repeat-containing protein 7. Feng et al (25) revealed that miR-25 promoted ovarian cancer cell proliferation and motility by directly 
targeting the serine/threonine-protein kinase LATS2. Zhao et al (26) reported that miR-25 enhanced the proliferation and motility of gastric cancer cells by inhibiting the expression of reversion-inducing cysteine-rich protein with Kazal motifs. The results of the present study indicated that the expression level of miR-25 was significantly increased in glioma tissues compared with normal brain tissues and that a high miR-25 expression level was significantly associated with advanced clinical stage. These data suggested that the upregulation of miR-25 may promote glioma progression. Additionally, miR-25 expression level was increased in glioma cell lines compared with normal brain tissues. Therefore, two glioma cell lines were transfected with a miR-25 inhibitor to decrease its expression. This revealed that the knockdown of miR-25 significantly reduced glioma cell proliferation, migration and invasion. Similarly, Zhang et al (22) showed that miR-25 was upregulated in $91 \%$ of human glioma tissues and four out of six human glioma cell lines examined and that miR-25 promoted glioma cell proliferation.

miRNAs function by regulating the expression of their target genes, a number of which have been identified for miR-25, including BTG2 (27), cyclic GMP-AMP synthase (28), transcription factor SOX4 (29) and CDKN1C (22). In the present study, a bioinformatics prediction showed that CADM2 was a putative target gene of miR-25. Luciferase reporter gene analysis further confirmed this targeting relationship. CADM2, a member of the synaptic cell adhesion molecule 1 family, contains three Ig-like domains and a cytosolic protein 4.1 binding site near the C-terminus, through which it crosslinks spectrin and interacts with other cytoskeletal proteins (30). It has been reported that CADM2 acts as a tumor suppressor in several common types of human cancer. He et al (30) reported that aberrant methylation and loss of CADM2 expression were associated with the progression of renal cell carcinoma. Furthermore, Yang et al (31) revealed that a low CADM2 expression level predicted a high risk of recurrence following hepatectomy in patients with hepatocellular carcinoma. The present study revealed that CADM2 was significantly downregulated in glioma tissues and cell lines compared with normal brain tissues. Moreover, a negative correlation was observed between the expression levels of CADM2 and miR-25 in glioma tissues and the expression of CADM2 was negatively regulated by miR-25 in glioma cell lines. These findings suggested that the increased expression level of miR-25 may contribute to a decrease in that of CADM2 in glioma. It was further revealed that the inhibition of CADM2 expression rescued the suppressive effects of miR-25 downregulation on the proliferation, migration and invasion of glioma cells, confirming that CADM2 acted as a downstream effector of miR-25 in glioma cells.

To the best of our knowledge, this is the first study to report that miR-25 promoted glioma cell proliferation, migration and invasion, at least in part by targeting CADM2. These findings expand the understanding of the underlying molecular mechanisms of glioma progression.

\section{Acknowledgements}

Not applicable.

\section{Funding}

This study was supported by grants from the Nature Science Foundation of Hunan province (no. 2021JJ70074).

\section{Availability of data and materials}

The datasets used and/or analyzed during the current study are available from the corresponding author on reasonable request.

\section{Authors' contributions}

CS designed the study and revised the manuscript. GP collected the clinical tissues, conducted the statistical analysis and wrote the manuscript. YL and CY performed the experiments. GP and CS have confirmed the authenticity of all the raw data. All authors have read and approved the final manuscript.

\section{Ethics approval and consent to participate}

The present study was approved by the Ethics Committee of Xiangya Hospital, Central South University (Changsha, China). Written informed consent was obtained from all participants.

\section{Patient consent for publication}

Not applicable.

\section{Competing interests}

The authors declare that they have no competing interests.

\section{References}

1. Torre LA, Bray F, Siegel RL, Ferlay J, Lortet-Tieulent J and Jemal A: Global cancer statistics, 2012. CA Cancer J Clin 65: 87-108, 2015

2. Siegel RL, Miller KD and Jemal A: Cancer statistics, 2015. CA Cancer J Clin 65: 5-29, 2015.

3. Marumoto T and Saya H: Molecular biology of glioma. Adv Exp Med Biol 746: 2-11, 2012

4. Goodenberger ML and Jenkins RB: Genetics of adult glioma. Cancer Genet 205: 613-621, 2012.

5. Ma Z: Downregulation of SETD 8 by miR-382 is involved in glioma progression. Pathol Res Pract 214: 356-360, 2018.

6. Tan Z, Zhao J and Jiang Y: miR-634 sensitizes glioma cells to temozolomide by targeting CYR61 through Raf-ERK signaling pathway. Cancer Med 7: 913-921, 2018.

7. Liu L, Liu Z, Wang H, Chen L, Ruan F, Zhang J, Hu Y, Luo H and Wen S: $14-3-3 \beta$ exerts glioma-promoting effects and is associated with malignant progression and poor prognosis in patients with glioma. Exp Ther Med 15: 2381-2387, 2018.

8. Peng Z, Liu C and Wu M: New insights into long noncoding RNAs and their roles in glioma. Mol Cancer 17: 61, 2018.

9. Dai S, Yan Y, Xu Z, Zeng S, Qian L, Huo L, Li X, Sun L and Gong Z: SCD1 confers temozolomide resistance to human glioma cells via the Akt/GSK3 $\beta / \beta$-catenin signaling axis. Front Pharmacol 8: 960, 2018

10. Che F, Xie X, Wang L, Su Q, Jia F, Ye Y, Zang L, Wang J, Li H, Quan Y, et al: B7-H6 expression is induced by lipopolysaccharide and facilitates cancer invasion and metastasis in human gliomas. Int Immunopharmacol 59: 318-327, 2018.

11. Kloosterman WP and Plasterk RH: The diverse functions of microRNAs in animal development and disease. Dev Cell 11: 441-450, 2006.

12. Zhang HD, Jiang LH, Sun DW, Li J and Ji ZL: The role of miR-130a in cancer. Breast Cancer 24: 521-527, 2017. 
13. Ambros V: The functions of animal microRNAs. Nature 431: 350-355, 2004.

14. Song H, Zhang Y, Liu N, Wan C, Zhang D, Zhao S, Kong Y and Yuan L: miR-92b regulates glioma cells proliferation, migration, invasion, and apoptosis via PTEN/Akt signaling pathway. J Physiol Biochem 72: 201-211, 2016.

15. Stojcheva N, Schechtmann G, Sass S, Roth P, Florea AM, Stefanski A, Stühler K, Wolter M, Müller NS, Theis FJ, et al: MicroRNA-138 promotes acquired alkylator resistance in glioblastoma by targeting the Bcl-2-interacting mediator BIM. Oncotarget 7: 12937-12950, 2016.

16. Zhu Y, Zhao H, Rao M and Xu S: MicroRNA-365 inhibits proliferation, migration and invasion of glioma by targeting PIK3R3. Oncol Rep 37: 2185-2192, 2017.

17. Zhang T, Ma G, Zhang Y, Huo $\mathrm{H}$ and Zhao Y: miR-599 inhibits proliferation and invasion of glioma by targeting periostin. Biotechnol Lett 39: 1325-1333, 2017.

18. Li Y, Tan W, Neo TW, Aung MO, Wasser S, Lim SG and Tan TM: Role of the miR-106b-25 microRNA cluster in hepatocellular carcinoma. Cancer Sci 100: 1234-1242, 2009.

19. Ding X, Zhong T, Jiang L, Huang J, Xia Y and Hu R: miR-25 enhances cell migration and invasion in non-small-cell lung cancer cells via ERK signaling pathway by inhibiting KLF4. Mol Med Rep 17: 7005-7016, 2018.

20. He J, Qi H, Chen F and Cao C: MicroRNA-25 contributes to cisplatin resistance in gastric cancer cells by inhibiting forkhead box O3a. Oncol Lett 14: 6097-6102, 2017.

21. Peng G, Yuan X, Yuan J, Liu Q, Dai M, Shen C, Ma J, Liao Y and Jiang W: miR-25 promotes glioblastoma cell proliferation and invasion by directly targeting NEFL. Mol Cell Biochem 409: 103-111, 2015.

22. Zhang J, Gong X, Tian K, Chen D, Sun J, Wang G and Guo M: miR-25 promotes glioma cell proliferation by targeting CDKN1C. Biomed Pharmacother 71: 7-14, 2015.

23. Livak KJ and Schmittgen TD: Analysis of relative gene expression data using real-time quantitative PCR and the 2(-Delta Delta C(T)) method. Methods 25: 402-408, 2001
24. Xiang J, Hang JB, Che JM and Li HC: MiR-25 is up-regulated in non-small cell lung cancer and promotes cell proliferation and motility by targeting FBXW7. Int J Clin Exp Pathol 8: 9147-9153, 2015.

25. Feng S, Pan W, Jin $\mathrm{Y}$ and Zheng J: MiR-25 promotes ovarian cancer proliferation and motility by targeting LATS 2 . Tumour Biol 35: 12339-12344, 2014.

26. Zhao H, Wang Y, Yang L, Jiang R and Li W: MiR-25 promotes gastric cancer cells growth and motility by targeting RECK. Mol Cell Biochem 385: 207-213, 2014.

27. Chen H, Pan H, Qian Y,Zhou W and Liu X: MiR-25-3p promotes the proliferation of triple negative breast cancer by targeting BTG2. Mol Cancer 17: 4, 2018.

28. Wu MZ, Cheng WC, Chen SF, Nieh S, O'Connor C, Liu CL, Tsai WW, Wu CJ, Martin L, Lin YS, et al: miR-25/93 mediates hypoxia-induced immunosuppression by repressing cGAS. Nat Cell Biol 19: 1286-1296, 2017.

29. Chen B, Liu J, Qu J, Song Y, Li Y and Pan S: MicroRNA-25 suppresses proliferation, migration, and invasion of osteosarcoma by targeting SOX4. Tumour Biol 39: 1010428317703841, 2017.

30. He W, Li X, Xu S, Ai J, Gong Y, Gregg JL, Guan R, Qiu W, Xin D, Gingrich JR, et al: Aberrant methylation and loss of CADM2 tumor suppressor expression is associated with human renal cell carcinoma tumor progression. Biochem Biophys Res Commun 435: 526-532, 2013.

31. Yang S, Yan HL, Tao QF, Yuan SX, Tang GN, Yang Y, Wang LL, Zhang YL, Sun SH and Zhou WP: Low CADM2 expression predicts high recurrence risk of hepatocellular carcinoma patients after hepatectomy. J Cancer Res Clin Oncol 140: 109-116, 2014.

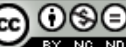

This work is licensed under a Creative Commons Attribution-NonCommercial-NoDerivatives 4.0 International (CC BY-NC-ND 4.0) License. 